\title{
Virtual reality in bioinformatics
}

\begin{abstract}
Virtual Reality (VR) is a well-known acknowledged field with many benefits in different sectors. There have also been many applications in entertainment, research, training and medical facilities. When we perform any action there is a similar reaction on real objects according to the nature hence it is a general understanding that we need to simulate the same action-perception relationship virtually without involving real objects or real events. Virtual Reality has a main component called the visualization that occurs by interaction between human and machine bringing it closer to real life. In virtual reality we need to break the barrier amid the real world and the virtual world. For the simulation of the technology one needs to produce an artificial sensation of touch, hear, smell, and feel based on the real one. In this paper we study about the different applications of Virtual Reality in Bioinformatics and also the history of Virtual reality. We take a brief look towards the immersive analytics of virtual reality. The tools used to produce the virtual environment are also being acknowledged with incorporating this knowledge. We also take a look towards the interaction of Virtual reality with many different properties and independent agents. We try to understand what a virtual world is and its uses. We illustrate the usage of Virtual Reality for cancer research and its approaches towards developing better medical therapies. Unfortunately, there are a few limitations that need to be overcome like affordability, unrealistic demonstration, and unawareness also discomfort while using the technology and affects to the eyes if used for a long time etc. It is also a very time consuming and complicated process in the making of the virtual environment. Facts like using normal input technologies like keyboard and mouse aren't very efficient for VR but also use of 3D glasses, tracking devices for movement like gloves and biosensors are very expensive to be bought by each individual.
\end{abstract}

Keywords: virtual reality, bioinformatics, artificial intelligence, immersive analytics, virtual environment
Volume 3 Issue 2 - 2019

Tanisha Batra, Pritee Chunarkar-Patil

Rajiv Gandhi Institute of IT and Biotechnology, Bharati Vidyapeeth Deemed University, India

Correspondence: Pritee Chunarkar-Patil, Rajiv Gandhi Institute of IT and Biotechnology, Bharati Vidyapeeth Deemed University, Pune, Maharashtra, India, Tel 9730038|42, Fax +9120-243657I3, Email preeti.chunarkar@bharatividyapeeth.edu, preetichunarkar@gmail.com

Received: March 14, 2019| Published: March 29, 2019
Abbreviations: VR, virtual reality; AI, artificial intelligence; VA, visual analytics; HDM, head-mounted displays; AL, artificial life; MDV, molecular dynamics visualization; PTM, post translational modifications

\section{Introduction}

Virtual reality (VR) is an influencing cyber- knowledge subject, occurring within an imitated environment that integrates mainly auditory and visual, but also additional kinds of sensory feedbacks. Virtual Reality linked topics play a main role in several research areas. Bioinformatics generally deals with biological macromolecules, the nominal creatures of existence; thus, visualization displays a main part in the study. The use of stereoscopic interpretation has remained for an extended time for envisioning molecular structures, coming reverse for the former buildup of VR equipment's was done before the CAVE was designed and Head-Mounted Displays (HMD) were available at a reasonable price. Generated by the accomplishment of novel, current HMDs like Oculus Rift as well as HTC Vive, Virtual Reality contains lately regained countless interest. Contrarily, a novel manufacture of significant "computational microscopes" has been recognized, for instance the CAVE2, producing outsized, just about 360 degree-spanning visualization services. Furthermore, in the earlier period these approaches were restricted to cost-intensive proficient visualization services. The arrival of fresh reasonably priced, and frequent mobile technologies, provides elevated probable for using related approaches on a regular basis for every day research. Visual Analytics is productively being used for numerous years to examine complex and heterogeneous datasets. Immersive Analytics combines these approaches now with new immersive and interactive technologies. ${ }^{1-3}$

Simultaneously, highly developed "Visual Analytics" clarifications have turned out to be a crucial instrument in all locale of technical information examination, uniting visualization, data mining along with investigation means with suitable customer interface. Fusing VR as well as VA, "Immersive Analytics" has begun to create novel techniques, engross the scientist hooked on the information, and, in the optimum instance, facilitates highly developed inherent. The present manuscript introduces a particular matter on this very topics. ${ }^{1}$ Investigation about virtual environments on one side as well as artificial intelligence furthermore has mainly survived because it has been accepted by two diverse communities with dissimilar obsession and interests, however a little union is currently noticeable among the two domains. Due to the union that has begun among the branches of complex calculations and exploring societies which were divided into sectors explicitly called Artificial Intelligence (AI) combined with Artificial Life (AL) and Virtual Reality (VR) can be considered as the driving force behind the thought of exhilarating functions. This mixture of intellectual skills and apparatus, personified in self-ruling beings and mediators, in cooperation among effectual resources for their graphical demonstration and interface of a variety of manner, has pre-arranged climb to an innovative region at their assembly end, which is currently experiential to be intelligent virtual environments. $^{4-22}$

Expertise comprises of a constituent of everyone's existence for a long time, along its contact with our standard of living has begun to enlarge mid instance. 'Virtual Reality' is a majorly 
pertinent, remarkable and lately strengthens expertise that has been put into practice in numerous approaches by now. Relating it to Structural Biology, virtual reality knowledge facilitates immersive 3D visualization of molecular arrangements, communications; machineries etc., in addition to molecular representation at the minuscule stage making possible an improved consideration of the science. This advancement helps in reading thoroughly and identifying malfunctioning origin grounds and expose efficient remedial treatments related to cancer. ${ }^{22}$ Even though this method has mainly involved a vast number of scientists and organizations, many fresh researchers and people everywhere starting to learn about virtual reality have very little or no knowledge about the practical benefits of this technology due to undersized disclosure of this field including the concern with expenses. It also mean to advise a novel combination of notion to supply to an superior advancement in cancer research upon which researchers can excellently submerge inside the integral atmosphere as well as come across responses by means of virtually interacting with some of the organisms that are invisible to the naked eye. This modifiable automation has been consequently, isolated as anticipated and acknowledged toward having immense potential moreover by now occurring in modernizing tutoring, guidance, technical study in addition to medicinal rehabilitation. ${ }^{23}$

\section{History}

Virtual Reality (VR), as the term states, it is theoretically supposed to be a replicated atmosphere which is not present in the actual world. Even though it is observed knowledgeably by a person using a peripheral head-gear exhibit and in commission with an explicit processor, it still presents a stinging sensation with the sensory coordination of the user's body as well as generates a realistic proposal with the intention that he/she is currently surrounded by artificiality deceived by what he/ she observes. Some characteristics of captivating quintessence of this automation gets into consideration for a single vital trademark in the domain of mechanization moreover has consequently aided humans to burn a bridge leading to an innovative method of investigation, progression and figuring. While the subsequent part of the decade, researchers progressed towards the establishment that amplified realism is not a mere fictional story anymore instead it is reasonably a multifaceted automatic setting established anticipated in support of an creatures to be aware in present instant. The past of 'Virtual Reality' signifies so as to its design with relevance in the actual humanity was stimulated by science fictional stories. ${ }^{1}$ In this age, by way of the augmented succession of mechanization, 'Simulated 3-D environment' have given the impression that it has percolated inside many features of expansion. Not just the gaming businesses formulate the employment of this automation widely in the current times however constant architectonical organizations, investigating groups, therapeutic and enlightening foundations and many more, give the impression of maintaining the impetus. Oculus VR, the major flourishing corporation to invent VR cast to be worn on the head like headphones revealed the creation of Oculus Rift (2016). Likewise, more globally accepted corporations enclosed some more advanced automations related to VR. Google advertised their merchandise 'Google Cardboard', a 'DIY' (Do It Yourself) receiver which requires a Smartphone to be slide within it to experience a simulated environment. Other companies like Sony, Samsung and HTC have also launched their inventions in the marketplace successfully. These products include captivated and facilitated an immeasurable amount of establishments and businesses crossways from the globe to imagine in a broader perspective plus identify ultramodern advantages. ${ }^{11}$
To take care of people enduring excruciating rehabilitation in opposition to melanoma, VR encloses a majestic ingression inside the domain as an unusual appearance of sickness to diminish psychological and substantial strain. Away from assisting in various therapeutic methodologies just to eventually be of assistance to victims tormented from tumor in addition to giving them expressive support with motivation, VR has now revealed immense prospective to increase bioinformatics study. The thought about visualizing, modeling or exploring a particle in a 3D area is a vast development balanced to burdening the identical predictable 2-D PC display. The prerequisite of networking among matter in the implicit situation through merely building physical signals plus not encompassing to inevitably wait in a single position constructs it to be greatly additional soothing, effortless, as well as quicker for a person to comprehend the surroundings moreover bear out the errands. By means of the following rights, VR has supplied us with the majority of advanced mode of conducting cellular studies at the structural point also it is certain that this automation is obtainable to pick up the scope of bioinformatics in the forthcoming years. ${ }^{23}$ Even though this influential automation directs towards revolutionary advancements to progress in undertaking and monitoring in the medicinal domain, present prolong hardly any constraints at hand. Some of them are elevated safeguarding expenses, soaring electricity utilization or else inadequate battery verve of the equipment, greatly extra-large gadgets, huge and non-functional wires, moral compulsion while managing victims' records also with use of recordable evidence by using Virtual Reality HMDs during therapeutic procedures. Distant as of definitely fitting a factor of a few observations extremely rapidly, it would be exposed towards being a dispute on the way to accept as well as believe that compensation under misconduct in every phase about medicinal concern. Although a few methodologies might contract the necessity designed for bothersome ropes along with bulky equipment's. ${ }^{4}$

In philosophy, Plato's Allegory of the Cave - published in his famous opus Republic - discusses one of the fundamental principles in discriminating realism, projections and the way the human being depends on his senses to understand the world. In this way, humans do not see the truth; they perceive reality, depending on their senses. Wherever there is a common denominator for all individual beings, there are sturdy personal differences, based on the civilization, socialization, and individual experiences. ${ }^{1}$ Virtual Reality now expands the projections of the real world to the digital world, a significant step in the mission for the ultimate display. ${ }^{2}$ The first HMD was developed by Sutherland around $1968 .{ }^{3}$ One of the first approaches to engage multiple senses of a viewer was even developed a few years earlier by Morton Heilig. ${ }^{4}$ In the 1980 s, the expression Virtual Reality became well-known, credit to pioneers like Jaron Lanier, who urbanized initial scientific devices sustaining the 3D visualization and communication with the computer. The adding up of haptics, as performed for biological applications, was a significant step. ${ }^{5}$ A big get through of the 1990s was the discovery of the CAVE®, the Cave Automatic Virtual Environment, and a visualization system consisting of three to six stereoscopic projection screens, which were installed at several research facilities worldwide. ${ }^{6}$ In Bioinformatics, many software applications were evolved to support VR-related technologies. Clearly, the visualization of the smallest entities of life, not observable to the naked human eye, is one of the application cases where the use of VR-related technologies is sensible and promising. Learning software like Meta!Blast, facilitate the stereoscopic visualization of 
group environments, goes a small footstep towards this course. ${ }^{7}$ By now in the premature time of the CAVE, researchers began to envision molecular structures in this atmosphere. ${ }^{8}$ Though, there was not a major breakthrough for this type of scientific visualization, because they were restricted to a few very cost-intensive research facilities. The arrival of new reasonably priced technologies in recent years may be able to alter this now.

\section{Immersive analytics in bioinformatics}

VR-associated approaches are visually imposing. In the appropriate case of spatial structures they, also, offer a precious extension of 3D visualizations to the bioinformatics area through their immersive character. Though, they are generally still distant from being very cooperative in supporting the analytics procedure. Visual Analytics was characterized by Thomas and Cook as "the science of analytical reasoning facilitated by interactive visual interfaces". ${ }^{9}$ To expand this approach to VR-related technology, the word Immersive Analytics was invented by Chandler et al.: "Immersive Analytics examines how new interaction and display technologies can be used to support analytical reasoning and decision making". ${ }^{10}$ A major characteristic of IA is typically the stereoscopic visualization that is used to submerge the user into the environment. ${ }^{11}$ In addition, IA expands these approaches to numerous senses, haptic as well as olfactory feedback are possible elements of these approaches. Even though the phrase is quite new, associated approaches were already developed numerous years ago. Software applications like MetNetVR included composite metabolic pathways/networks hooked on theoretical unit locations furthermore envisaged it into the CAVE intended for methodical reasons around $2006 .{ }^{12}$ For navigation plan, a tablet was used. By means of the CELL microcosms Pathway combination, metabolic pathways are capable of partially being entrenched in the virtual unit surroundings furthermore evaluated in discrete virtual environments, such as zSpace ${ }^{\circledR}$ and/or CAVE2 ${ }^{\circledR} .{ }^{11-13}$ The zSpace ${ }^{\circledR}$ is a semi-immersive stereoscopic display tool which can be used for head-tracked stereoscopic visualization, allowing the display perspective to be modified because the person shifts in front of the demonstration. 3D interaction is achievable with a special stylus pen. This arrangement facilitates hybrid-dimensional visualization and interaction, visualizing spatial structures with abstract data side-by-side. ${ }^{14}$

\section{Applications of virtual reality in bioinformatics}

HoloLens: Automation for visualizing graphically interacting molecules

A significant device in the field of Augmented Reality applications is the Microsoft HoloLens ${ }^{\circledR}$. This device can be used to position virtual substance into real-world environments. The document shows proteins floating over a table or projected in front of a tree. In this method, the observer can examine the spatial arrangement of the protein from all perspectives by means of natural displacement and body movement. Related technologies may be used in forthcoming approaches to elaborate protein structures collaboratively in the perspective of a regular environment, detached from the finite prospect arranged by regular computer displays. In case of the HoloLens, the big benefit of an incorporated computing device comes with a disadvantage: the computer presentation is quite little. In case of molecular data, where usually thousands to millions of atoms are visualized as spheres, this restraint is challenging. In this work, the performance of the HoloLens is investigated, elongated by a good overview regarding related application cases. A natively developed Universal Windows Platform application with diverse rendering techniques is compared to a Unity application in conditions of performance, changing the number of atoms to be visualized. ${ }^{15}$

\section{3-D Stereoscopic recreation of microscopic organisms}

Chlamydomonas reinhardtii is a unicellular organism which plays a significant part in the field of metabolic engineering as it can be used as a substitute energy source. In this work a spatial mesoscopic model of this cell was formed using an interpretative cell visualization approach. The plan is to unite the knowledge of diverse information sources - such as publications, microscopic images, and database information - with the principle to form a well-evaluated cell model which can be used for visualization and exploration purposes. The so-formed model was used as a source for a stereoscopic 3D cell animation, discussing the primary metabolic procedures to pull out energy from a biological cell. The publication discusses the nature of the diverse information sources, the design choices concerned in the procedure (e.g. in terms of coloring, texturing and structure building), the manner the flagella-based movement of the cell was animated, as well as the particular necessities to generate a stereoscopic 3D animation. The primary observations are relevant for content formation for virtual environments and should support visualization researchers to use stereoscopic 3D visualization in the context of their work which also plays a significant role in Virtual Reality-related applications. ${ }^{16}$

\section{Use of virtual reality in structural biology and molecular modeling}

A main field in Bioinformatics as well as Chemo informatics research is the simulation of molecular structures. One of the majorly recognized techniques is the molecular dynamics (MD) simulation approach. Such simulations were executed in the context of this work with the Gromacs software. ${ }^{17}$ The visualization of molecular dynamics has a lengthy custom, by means of standard tools such as VMD or PyMol. Though, these visualization packages are not advanced to be used in the context of multiscale virtual 3D stereoscopic environments. The molecular dynamics visualization (MDV) software has been developed on top of the Unity development stage and can be used with a variety of large-scale immersive displays. The HIVE Cylinder at Curtin University for example consists of a 3 $\mathrm{m}$ high $\times 8 \mathrm{~m}$ diameter screen with a $180^{\circ}$ field-of-view. The screen is illuminated using three $1920 \times 1200$ DLP projectors deformed and blended to provide a nonstop image across the screen, supporting 3D stereoscopic visualization with (active) liquid-crystal shutter 3D glasses. Using MDV, MD simulations can be collaboratively as well as immensely inspected with groups of up to 50 people. Standard molecular visualization metaphors, such as ball \& stick and secondary structure representations are enforced and can be chosen via a 3D user interface. ${ }^{18}$

\section{Minomics: A centralizing and mesmerizing gadget for multiple-omics exploration}

MinOmics is a unified analysis pipeline and visualization framework for multi-omics analysis. In this work, a stereoscopic 25 MPixel display wall was used to display proteomic information on 1417 proteins of Chlamydomonas reinhardtii. For this reason, UnityMol was used, a Unity $3 \mathrm{D} \AA$-based software function which visualizes proteins in 3D. ${ }^{19} \mathrm{~A}$ number of hardware setups are considered which could be used with MinOmics: a UnityMol-based 
WebGL and WebVR function using a usual monitor, a split-screen approach united to a stereoscopic monitor, and a full-immersive environment using head-mounted displays. A number of scientific application cases for these setups are discussed. For example, the MinOmics framework can be used to discover multiple omics datasets. The authors converse the investigation of redox proteomic datasets in the context of Chlamydomonas reinhardtii for glutathionylation, nitrosylation and disulphide bonds. MinOmics can be used to discover protein specificity of multiple redox post translational modifications (PTM), the cysteine specificity of multiple redox PTMs, and to discover structural determinants of redox-modified cysteines. ${ }^{20}$

\section{Visualization and analysis of molecular data using HIVE}

Most bioinformatics-related workflows need the use of numeral diverse tools. Frequently this diversity means that information has to be changed, transformed to other formats and transferred leading to a vast overhead of time and expenses. This overhead applies particularly to virtual environments, where at times the information to be entirely visualized has to be optimized by visualization experts to formulate it well-suited to the virtual environment, such as the abovementioned HIVE Cylinder display. In this work, an integrated pipeline is introduced, promoting straight interaction on semantically-linked 2D and $3 \mathrm{D}$ heterogeneous data, which is completely displayed in a virtual working place. The focus here is on the interactive visualization of heterogeneous molecular data which is united based on accessibility or conditional relations following hierarchical concept definitions. The user is supported by the developed system with adaptive analyzes modified towards the required tasks. The developed ontology describes both structural biology as well as interaction concepts. ${ }^{21}$

\section{Virtual environment gears}

The fusion of procedures essential to run the following fusion of sophisticated mechanisms proposes so as amid the growth of additional complicated schemes, gears also matured settings are capable of taking part in an essential part of the development in the domain. Presently there is supposed to be quite a lot of diverse affairs to think, concerned with the stage of generalization of this hold up, information depiction furthermore the combination of composite properties. $^{22}$

\section{The advancement of virtual environment}

The advancement of intellectual VEs is still more forced through the partiality of the majority usually accessible VE toolkits approaching illustrated practicality as well as the diagrammatical sustenance of the VE, relatively better than approaching the accumulation of brainpower. At the simplest stage, a scheme may exist advanced with, for instance, Open GL, otherwise several additional 3D collection scheme as well as $\mathrm{C}++$ however, as customary, elasticity is bought-off opposing to the occasion and attempt crucial to achieve the favored purpose. A distinct illustration of VE advancement at the simplest point is the $\mathrm{AReVi}$ toolkit ${ }^{23}$ which offers a set of $\mathrm{C}++$ lessons built around an agent programming language. The novel junction of appeal represents, VE toolkits exploit the scene-graph illustration beforehand referred. This is quite an appropriate method of depicting the diagrammatical form of substances, like leaf nodes in the picture chart usually symbolize graphical primordial items the same as a set of hexagons. Thus primitives are subsequently organized into additional composite diagrammatic things by means of collection of points to whom the participants are adhered. ${ }^{23}$

\section{Relations of virtual reality through composite properties}

Proviso we desire to put together supplementary composite properties then illustrated manifestation towards items within a VE, an additional concern occurs linking through the customs where an entity plus a VE interrelate. ${ }^{24}$ Illustration contact in these traditional intellects acquires position basically in the midst of a VE entity along with the VE client, as well as covered by the entire image demonstration about an entity towards the client, together with consistency, illumination possessions and rank of specificity. Within a typical VE, items simply work together visually in the midst of every extra insofar as a single conceals one more as of the client's visualization. ${ }^{25}$ On the other hand, again supplementary composite properties are added, the quantity of relations linking VE items with surrounded by matter plus the VE itself augments orderly. An issue that requires dealing with is how extreme these contacts ought to exist determined through the entity moreover via the surroundings within which the entity is sited. So far, there isn't an apparent declaration of this difficulty. An individual advancement is to implant, within the entity and the properties and the essential information of the way we cooperate in the midst of a surrounding. For instance, the IMPROV method $^{25}$ approves what Goldberg identifies as inverse causality, as well as supplies simulations about the interrelation involving an entity plus a implicit creature surrounded through the entity, in regulation to eliminate a few insight obligation from the implicit creature. Therefore, an implicit creature that spots a virtual bottle of water is specified the substitute of consumption as of it devoid of including to be trained with the critical measures. This seems a tiny counterintuitive while bearing in mind the assimilation of features of physics such as gravity ${ }^{26}$ into a VE. At this point, it seems preferable that each and every one of the objects positioned within a VE should follow whatsoever substantial laws exist, suppose it has been declining downwards but not backed up for solemnity, otherwise hovering for non-solemnity. An instance about the similar kind might be why a fish must act if it's positioned in a place that isn't filled with water. ${ }^{24}$ Solitary way may perhaps exist to contribute properties like energy, permitting an entity to interrelate within a reasonable technique in the midst of several VE where it is supposed to be entrenched. It appears obvious so as the effort during AI lying on general ontology's could contain an essential function at this point. ${ }^{26,27}$

\section{Independent (autonomous) agents}

The union involving $\mathrm{AI}$ and $\mathrm{AL}$ together in addition to VEs lying on the supplementary is not found as understandable since within the vicinity of agents. These agents, like a investigate domain has break open inside a agitation of commotion in the period of most recent 5 years otherwise subsequently as designated, for example via the escalating amount of teachings and symposiums, in addition to the huge amount of dynamic study assembly. ${ }^{26}$ We differentiate now among self-sufficient agent investigate as well as extra wide-ranging place consisting of multi-agent arrangements. ${ }^{28,29}$ The final region encompasses scattered analytical submissions which include complex administration, which don't classically engage virtual environments also along with a focal point on internal contact plus cooperation to might not be necessary in the self-governing agent's subordinates. We have added about artificial managers, implicit creatures, virtual beings, moreover personifications (like user) inside 3-D multi-user network environments. We begin through a reflection about the natural world and task of sovereignty, also afterwards inspect the variety of causes, by means of sentiment, like a method of discriminating them. In each 
and every thing this, we argue effort amid an early summit in section of VE's concerned with job commencement related to concerns in AI.

\section{Virtual worlds}

If an immense arrangement of effort within the domain of VE is stirring near intellectual virtual environments in arrangement to attach explicit portions of behavior, whereas attempt in Artificial Intelligence considers the exercise of $\mathrm{VE}$ as a way of generating additional fascinating intellectual agents. These agents consist of the formation of virtual worlds containing digital life, absolute resemblance to persons in the actual world. Several observe dispersed useful virtual environments such as Dynamic foundation intended for the improvement of parts of the virtual worlds, on condition that a place for the examination of AL as well as of ancient independent contact among AL as well as virtual worlds. Wherever lot of work into genetic algorithms resides over the position of genotype in which a virtual world could authorize the investigation of the phenotype at the penalty of a meticulous hereditary dataset along amid the study of progression about the phenotype intensity in the equivalent variety of approach as represented in the real world..$^{22} \mathrm{~A}$ paradigm of this advance to VEs is the Nerve Garden scheme, ${ }^{30-32}$ which is a client-server coordination that agrees consumers to produce 3D plant replicas by means of L-systems (Lindenmayer Systems).$^{33} \mathrm{~A}$ client can subsequently pick a fastidious plant furthermore position it inside a plot in a VRML island patch VE. A variety of viewpoints were presented, together with a vibrant perspective starting as of the backside of an airborne bug animatronics, which looped incessantly beyond the desert island. Resonance special echo effects were supplementary, together with rumbling sounds of rumble, which escorted L-system supported effective lightning. The effort is practically primordial as of an AL viewpoint in view of the fact that it doesn't sustain plant development otherwise relations among plants as well as surroundings, nevertheless a Nerve Garden II venture endeavors to assemble this insufficiency through increasing a straightforward but valuable plot ecological unit. ${ }^{22}$

\section{Virtual reality - approach in cancer research}

A few Scientists considered the worth of VR throughout the preparation of sections of tumors in the province of 50 participations. In few scenarios, VR skills facilitated brain surgeons by means of 3-D descriptions about the framework in the area subsequent to every patient undergo head CT scans. These statistics offered as a result of the VR method are written also used as orientations by the fitness interior throughout surgeries. ${ }^{34}$ Another scientist asserted about a procedure, machine support pre-surgical preparation or else 'CAPP', assisted towards progress of 3-D practical smearing of anatomic sampling with depiction of malignant tumors, significant operative segments, boundaries. This procedure is able to subsist to use to accumulate the in order in a meticulous arrangement also afterward exist joint among oncologists and pathologists, inside arrangement towards exclusively exemplify the collected tumor records. ${ }^{35}$ People speak regarding another bright advancement commenced by a scientist wherever VR is measured a lot improved substitute to identify tumor related to patients in contrast with conventional scientific observations that engage illustration events by means of potentially injurious emission which might amplify the threat of melanoma. ${ }^{36}$ 'Ross' (Robotic Surgical Simulator) is a virtual simulator used to train aspiring surgeons and was developed in 2011. This invention alarmed an assembly that incorporated doctors qualified to perform mechanized operations. Many of the surgeons powerfully believed on the invention and helped to incorporate this for teaching as well as carry out trials on a person's proficiency prior to the contribution they made during the authentic procedure, but some contemplation about the narrative innovation was in no shape to practice it in mechanized surgical procedure ${ }^{37}$

Speaking about the similar perception, a researcher made his statement about teaching or producing doctors having mechanized methods, with way of Virtual mechanized simulators prior to performing surgical treatment, are essential in India due to lack of doctors and increasing population. ${ }^{38}$ There were guidebooks created for brain-cancer surgeries which included use of VR simulators like NeuroTouch ${ }^{39}$ 3D VR simulations with the Dextroscope method ${ }^{40}$ otherwise 3D anatomical learning of tumors, ${ }^{41}$ it began to be essential to have worldwide learning and guidance methods ought to initiate maintenance swiftness with such expertise in sort to develop physical performance throughout pre-surgical and surgical procedures. ${ }^{42}$ Even though this influential knowledge demonstrate the method to ultramodern advances to improve training and perform in the surgical field, here persist a small amount of restrictions at hand such as elevated maintenance expenses, soaring power utilization or inadequate sustaining the machines greatly extended gadgets, huge with wires that were not easy to use or handle, moral commitments whilst managing patient information via recordable VR HMDs for the period of remedial practice etc. separately on or after unquestionably fitting a fraction of the following almost immediately, it would go round for a dispute whether to undertake as well as believe about the compensation more than the problems in every facet of the therapeutic heed. ${ }^{43-46}$ However a few procedures might get rid of the unhandy wires with weighty equipment. At this point, the user is interrelating among the information plus studying it in their individual illustration viewpoint after wearing a HMD along with a data glove. Though it is revealed that this technology has not been dynamically disguised clinically so far, it does propose likelihood to get better and optimized medicinal functioning development and guidance ${ }^{47}$

An additional straightforward and well-organized method through which malignancy victims are promoted engages merely through the use of a VR headgear in addition to a pair of headphones to be worn for sound effects. Thus commences as well as assists few of the character contribute that is preferred effective surroundings \& by this means diverges his/her intellect as of the disagreeable bodily ambience, supporting in their psychosomatic and physical patience next to troublesome healing and conquer distressful indications. ${ }^{48}$ This method which automatically replicates a disconnected visual world as well activates the intellect of feel and inquiry equivalent to the amplified representation that the individual is interrelating with. ${ }^{49}$ Therefore, it will be capable of inspire the discharge of 'pleasant hormones' in cancer patients accordingly diminishing bodily and psychological trauma ${ }^{50}$ Another Researcher states, due to technological investigation with manifestation, VR incorporated dropping exhaustion as well as the effects whilst melanoma-patients endure treatment, plus the people that practiced appeared to be featured less significant harmful emotional indicators also enhanced psychologically ${ }^{51}$ Nevertheless, scientists affirmed so as to though people pronounce to facilitate treatment healing appear smaller and enhanced due to understanding VR in use entertainment interference, this conclusion established the fact About VR merely assists sickly people to endure the treatment and not essentially advance signs connected with chemotherapy. Additionally, patients being capable to at the same time take pleasure in as well as endure the indications extremely healthily, and making VR less expensive for entertainment method intended for treatment are a small number of foundation when 
is optional though it is supposed to have been executed therapeutically as a cure to the people who will be affected by distress. ${ }^{51,52}$

\section{Future aspects of virtual reality}

The immense difficulty in scheming current civilization is if VR will be able to now continually extend to a degree or it will possibly will authentically substitute reality ${ }^{53}$ so that we utilize the knowledge to cooperate with equipment and carry out errands logically more competently. Like the ordinary items that have been observed along with interrelate amid often through the actual universe, now VR had drastically verified to them with the intention of doing the same. It has been anticipated that this technology is going to turn into an enormous style and obtain above our lives in the future. However, the further demand is the interpretation of existence based on Biological with chemical intensity related to actuality. Separately based on predictable medicinal exploration lessons, the following apparatus is related to physically powerful prospective that widely supports scientists about not directly assisting people struggle against melanoma. A group of scientists have accomplished that it is possible that we can practically travel inside an existing cell also yet appear within it and learn the internal organelle system with just a pair of earphones, headgears and controlling our movements with gadgets like joystick, game control etc. This was accomplished by travelling through diverse positions in some tumors superficial exterior by means of a key along with show a besieged nano-particle contracts being engrossed inside a cell. Furthermore, there have been trials to devise such machinery that are intended to discharge smaller medicines, position such learning skills about the molecular possessions to make medicine by changing its dimensions through simulations and thus has applications in developing machines to increase drug consumption, supporting drug discovery, enduring sarcoma treatments..$^{54,55}$

Use of VR for educational purposes can be done by learning chemical constitution and its familiarity to the biological area. For example, people are now able to precisely tour indoors into the blood vessels of their own body plus monitor magnitude as well as locality of cholesterol accretion as an alternative to make rigid recognition of a representation is a conservative technique. ${ }^{57}$ The technology once made available would actually encourage people to do their own medical checkups without wasting much time and money. Furthermore, supplementary hopeful views to make an optimistic collision about the technical discovery with providing researchers the statistics starting a novel viewpoint. ${ }^{56}$ Researchers have continuingly utilized VR to smooth undoubtedly revise plus understand transformed DNA accountable to produce malignancy. VR can help to envision and relate to the structures of proteins in an environment with stimulation. This technique will help us to recognize the hereditary reason for melanoma as well as in hospitals along with clinics in additionally decoding a solution to the most favorable cure consequently. It is believed, that the charges for VR apparatus will ultimately go down and each and every doctor or human being will possess the apparatus for such treatments in a short duration of time. ${ }^{58}$

\section{CAVE:An automatic virtual environment}

It is a future three dimensional industrial technology. It was written in java. It advances from beginning to end which one single will be capable of carrying out bimolecular research. Being a substantial proposal that sends the client to a material space that allows him/ her to stroll about as well as relate its fundamentals to the real world devoid of conservative hearing although by using a LED spectacle that present an outcome to the user..$^{59,60}$ The displays assist the user envisage this space also share this similar experience with one more being concurrently. This technology aspires towards conquering demerits of HMDs. CAVE2 had a more devoted intent of serving scientists envision their information efficiently. ${ }^{61}$ CAVE used MD models for studying the impending tumor protein structures with its behavior and many other factors. Spectators might investigate particles and structures next to controlling them plus rotating it. Occasionally, towards improving its understanding, there was a need to develop the virtual environment completely, progress seen with the blueprint using magic virtually with the use of a wand controlled with game consoles also undertake strict remembrance restrictions. ${ }^{62,63}$ Nowadays, still the organization of Weill Cornell Medicine makes use of CAVE to effortlessly envision remedial sets through the improved description of the technology. This definitely approaches to realistic sense of a variety of sectors counting it to revise the body structure components through the worldwide altitudes of the technology subsequent in pictorial view are acquired from CT. ${ }^{64}$ It has been declared significant to converse to another group of beings simultaneously as studying the particle in VR as an alternative of doing something in the given space while envisioning along with, therefore desire expansively utilize this technology by actual probability, plus concurrently converse with other natives while the fragment glide in 3D space in front of them (Figure 1). ${ }^{65-74}$

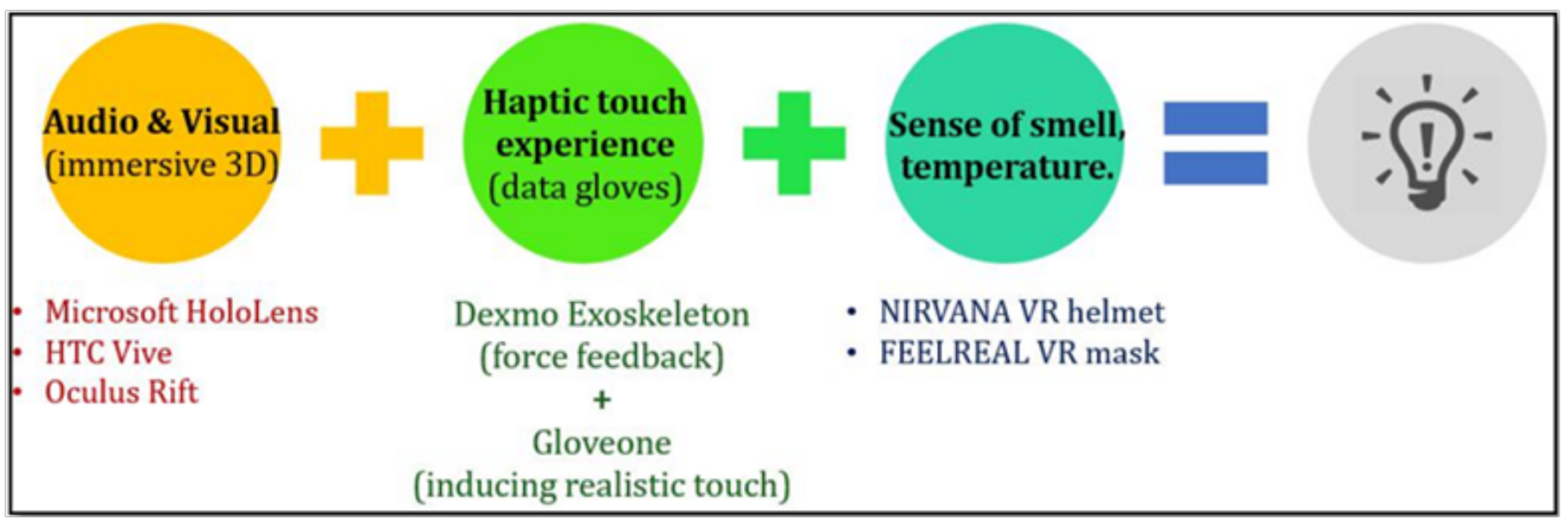

Figure I Creating a new idea by combining some or allVR technologies. ${ }^{67}$ 


\section{Conclusion}

Virtual Reality has been with us for a long time now but this technology is unknown to many people till date. There have been some changes lately; the use of VR has reached to a advanced level like in schools and colleges, medical treatments, research facilities and many more. It has mainly helped in cancer studies not directly curing it but helping us find a way around the treatments. The products designed for the use of VR will soon become easily available and affordable to many people and help in making this technology bigger and better. People need to be more involved in spreading the advantages of this technology including young minds with better ideas to finally make our lifestyle more advanced and improve society in many places around the globe. Research till this date determines that this technology can soon be available to a number of hospitals, clinics, medical examiners, etc to explore the disease and discuss this with more visibility inside the body by just wearing a pair of glasses and headphones. With this overview it is our hope to encourage more Bioinformatics-related exploration into Virtual Reality and its relation with artificial intelligence in the future. I hope that in future projects this technology will be utilized, offering unlimited opportunities for exploring virtual reality, where it has not been possible to be used in other fields.

\section{Acknowledgments}

Undertaking a review on "Virtual Reality in Bioinformatics" and submitting detailed dissertation would not have been possible without the remarkable guidance and support of my review guide Mrs. Pritee Chunarkar-Patil, Department of Bioinformatics, Rajiv Gandhi Institute of IT and Biotechnology, whose valuable contributions paved my way towards completing of my review. I am highly grateful and obliged to her for her dedicative support. I wish to express my profound sense of gratitude to Dr. S. Shaikh, Principal, Rajiv Gandhi Institute of IT and Biotechnology, for kind blessing and encouragement. I would like to thank my parents for their motivation and encouragement during completion of my review.

\section{Conflicts of interest}

The author declares there is no conflict of interest.

\section{References}

1. Björn Sommer, Marc Baaden, Michael Krone, et al. From Virtual Reality to Immersive Analytics in Bioinformatics. J Integr Bioinform. 2018;15(2):20180043.

2. Sutherland IE. The ultimate display. Multimedia: From Wagner to virtual reality. 1965 . p. 506-508.

3. Sutherland IE. A head-mounted three dimensional display. In Proceedings of the December, 9-11, 1968, fall joint computer conference, part I. ACM. 1968:757-764.

4. Heilig ML. Sensorama simulator. US Patent 3,050,870, Google Patents. 1962.

5. Brooks Jr FP, Ouh-Young M, Batter JJ, et al. Project GROPEHaptic displays for scientific visualization. In: ACM SIGGraph computer graphics. ACM. 1990;24:177-185.

6. Cruz-Neira C, Sandin DJ, DeFanti TA, et al. The CAVE: audio visual experience automatic virtual environment. Commun ACM. 1992;35:64 72 .

7. Wurtele ES, Bassham DC, Dickerson J, et al. Meta!Blast: a serious game to explore the complexities of structural and metabolic cell biology. In: Proceedings of the ASME 2010 World Conference on Innovative Virtual Reality. ASME. 2010;237-240.
8. Cruz-Neira C, Langley R, Bash PA. VIBE: a virtual biomolecular environment for interactive molecular modeling. Comput Chem.1996;20(4):469-477.

9. Cook KA, Thomas JJ. Illuminating the path: the research and development agenda for visual analytics. Richland WA (US): Pacific Northwest National Laboratory (PNNL). 2005.

10. Chandler T, Cordeil M, Czauderna T. Immersive analytics. In: Big Data Visual Analytics (BDVA). IEEE. 2015;1-8.

11. Sommer B, Hamacher A, Kaluza O, et al. Stereoscopic space map semi-immersive configuration of $3 D$-stereoscopic tours in multi-display environments. In: Electronic Imaging, Proceedings of Stereoscopic Displays and Applications XXVII. 2016;2016:1-9.

12. Yang Y, Wurtele ES, Cruz-Neira C. Hierarchical visualization of metabolic networks using virtual reality. In: Proceedings of the 2006 ACM international conference on virtual reality continuum and its applications. ACM. 2006;377-381.

13. zSpace. zSpace Aesthetics. 2015.

14. Sommer B, Wang SJ, Xu L. Hybrid-dimensional visualization and interaction-integrating $2 D$ and $3 D$ visualization with semi-immersive navigation techniques. In: Big Data Visual Analytics (BDVA). IEEE. $2015 ; 1-8$.

15. Müller $\mathrm{C}$, Huber $\mathrm{M}$, Biener V, et al. Interactive molecular graphics for augmented reality using HoloLens. $J$ Integr Bioinform. 2018;15(2):20180005

16. Biere N, Ghaffar M, Doebbe A, et al. Heuristic modeling and 3D stereoscopic visualization of a Chlamydomonas reinhardtii cell. $J$ Integr Bioinform. 2018;15(2):20180003.

17. Hess B, Kutzner C, van der Spoel D, et al. Gromacs 4: algorithms for highly efficient, load-balanced, and scalable molecular simulation. $J$ Chem Theory Comput. 2008;4(3):435-447.

18. Mancera R, Wiebrands M, Malajczuk C, et al. Molecular dynamics visualization (MDV): stereoscopic 3D display of biomolecular structure and interactions using the Unity game engine. J Integr Bioinform. 2018;15(2):20180010.

19. Doutreligne S, Cragnolini T, Pasquali S. Unity Mol: interactive scientific visualization for integrative biology. In: Large Data Analysis and Visualization (LDAV), 2014 IEEE 4th Symposium on. IEEE. 2014;10910 .

20. Maes A, Martinez X, Druart K, et al. MinOmics, an integrative and immersive tool for multi-omics analysis. J Integr Bioinform. 2018;15(2):20180006.

21. Trellet M, Férey N, Flotyński J, et al. Semantics for an integrative and immersive pipeline combining visualization and analysis of molecular data. J Integr Bioinform. 2018;15(2):20180004.

22. Ruth Aylett, Michael Luck. Applying Artificial Intelligence to Virtual Reality: Intelligent Virtual Environments. Applied Artificial Intelligence An International Journal. 2000;14(1):3-32.

23. Reignier P, Harrouet F, Morvan S. Arevi: A virtual reality multiagent platform. In Heudin JC editor. Virtual Worlds. 1998. p. 229-240.

24. West A, Hubbold R. Research challenges for systems supporting collaborative virtual environments. In Proceedings, Collaborative Virtual Environments. 1998. p. 98.

25. Goldberg A. Improv: A system for real-time animation of behaviorbased interactive synthetic actors. In Trappl R et al., editors. Creating Personalities for Synthetic Actors. 1997. p. 58-73.

26. Aylett R, Brazier F, Jennings N, et al. Agent systems and applications. Knowledge Engineering Review. 1998;13(3):303-308.

27. VRML Consortium. Living worlds: Making VRML 2.0 applications interpersonal and interoperable, draft 2. 1998. 
28. Luck M. Foundations of multi-agent systems: Issues and directions Knowledge Engineering Review. 1997;12(3):307-308.

29. Luck M, d'Inverno M, Fisher M, et al. Foundations of multi-agent systems: Techniques, tools and theory. Knowledge Engineering Review. 1998;13(3):297-302.

30. Sims K. Evolving 3d morphology and behaviour by competition. Artificial Life. 1995;1(4):353-372.

31. Prophet J. Technosphere. Interpretation. 1996;2(1).

32. Damer B, Marcelo K, Revi F. Nerve garden: A public terrarium in cyberspace. In Heudin JC editor. Virtual Worlds. 1998. p. 177-185.

33. Prusinkiewicz P, Lindenmayer A. The Algorithmic Beauty of Plants. Springer-Verlag. 1990.

34. Wang S, Zhang S, Jing J. Stereoscopic virtual reality models for planning tumor resection in the sellar region. BMC Neurology. 2012;12:146.

35. Essig H, Rana M, Meyer A, et al. Virtual 3D tumor marking-exact intraoperative coordinate mapping improve post-operative radiotherapy. Radiat Oncol. 2011;6:159.

36. Zanzonico P. Virtual Reality for Dose Optimization in Pediatric Nuclear Medicine: Better Than the Real Thing. Journal of Nuclear Medicine. 20111;52(12):1845-1847.

37. Seixas-Mikelus SA, Stegemann AP, Kesavadas T, et al. Content validation of a novel robotic surgical simulator. BJU International. 2010;107:1130-1135.

38. Rajanbabu A, Drudi L, Lau S, et al. Virtual Reality Surgical SimulatorsA Prerequisite for Robotic Surgery. Indian J Surg Oncol. 2014;5(2):125127.

39. Alotaibi FE, AlZhrani GA, Mullah MA, et al. Assessing Bimanual Performance in Brain Tumor Resection with NeuroTouch, a Virtual Reality Simulator. Neurosurgery. 2015;11(Suppl 2):89-98.

40. Shi J, Xia J, Wei Y, et al. Three dimensional virtual reality simulation of periarticular tumors using Dextroscope reconstruction and simulated surgery: A preliminary 10-case study. Medical Science Monitor. 2014;20:1043-1050.

41. Swanson K, Alvord E, Murray J. Virtual brain tumours (gliomas) enhance the reality of medical imaging and highlight inadequacies of current therapy. British Journal of Cancer. 2002;86(1):14-18.

42. Lendvay T, Hannaford B, Satava R. Future of Robotic Surgery. The Cancer J. 2003;19:109-119.

43. How Is Stomach Cancer Diagnosed? 2016.

44. Ahlberg G, Hultcrantz R, Jaramillo E, et al. Virtual Reality Colonoscopy Simulation: A Compulsory Practice for the Future Colonoscopist? Endoscopy. 2005;37(12):1198-1204.

45. Mohammed A, Simpson A, Zamora I, et al. Virtual cystoscopy. Expert Review of Molecular Diagnostics. 2008;8(4):449-454.

46. Khor WS, Baker B, Amin K, et al. Augmented and virtual reality in surgery-the digital surgical environment: applications, limitations and legal pitfalls. Ann Transl Med. 2016;4(23):454.

47. Lin $\mathrm{Q}, \mathrm{Xu} \mathrm{Z}, \mathrm{L}, \mathrm{B}$, et al. Immersive virtual reality for visualization of abdominal CT. Proceedings of SPIE International Society for Optical Engineering. 2013.

48. Schneider S, Hood L. Virtual Reality: A Distraction Intervention for Chemotherapy. Oncol Nurs Forum. 2007;34(1):39-46.

49. Schneider S, Workman M. Virtual reality as a distraction intervention for older children receiving chemotherapy. Pediatr Nurs. 2012;26(6):593597.

50. Oyama H. Virtual reality for palliative medicine. Studies in Health Technology and Informatics. 1998;58:140-150.
51. Mary Dacuma. New virtual reality program for young cancer patients aims to lift their spirit. 2016.

52. Schneider S, Kisby C, Flint E. Effect of virtual reality on time perception in patients receiving chemotherapy. Support Care Cancer 2010;19(4):555-564

53. Will virtual reality replace physical reality? - Culture, Economics \& Politics of the Future. Future Timeline forum. 2016.

54. Identifying and understanding user groups for virtual reality (VR) medical visualizations-John McGhee. 2017.

55. Strom M. Virtual reality trip inside cancer cells can help design better chemotherapy drugs. The Sydney Morning Herald. 2016.

56. Bogle A. Virtual reality allows scientists to walk into a cancer cell. Mashable. 2016.

57. Virtual reality lets you stroll around a breast cancer cell. New Scientist. 2016.

58. Here's How Virtual Reality Could Help Doctors Treat Cancer. 2016. Popular Science. 2016.

59. Bio-IT World. Bio-itworld.com. 2005

60. Visbox, Inc. CAVE Systems. 2016. Visbox.com. 2016.

61. Tougaw D, Will J. Visualizing the Future of Virtual Reality. Computing in Science \& Engineering. 2003;5(4).

62. Hagenberger ME, Johnson P, Will J. Virtual Engineering Education - The Next Step In Bringing Technology To The Classroom. Indiana University Purdue University Fort Wayne (IPFW). 2006. p.1-9.

63. Cruz-Neira C, Jason Leigh, Michael Papka, et al. Scientists in Wonderland: A Report on Visualization Applications in the CAVE Virtual Reality Environment. Electronic Visualization Laboratory, University of Illinois at Chicago. 1993. p. 1-8.

64. Ahsan K. Visualizing Protein Structures in Virtual Interactive Interface. 2015. p. 1-4.

65. Heilmann C. My visit to the medical Holodeck - cancer research at Weill Cornell using HoloLens and the VR Cave. Christianheilmann.com. 2017.

66. Michael Zyracki, Andrew Kimoto, Malte Tinnus, et al. 3D Visualization Of Molecular Data Using A Cloud-Based Streaming Framework For Web-Based Large Model Viewing. Autodesk Research. 2015.

67. Dheeraj Prakaash, KR Ravikumar, Mallesha H. Virtual Reality: A Railroad for Structural Bioinformatics towards 1 Advanced Cancer Research. Research and Development Centre, Robust Materials Technology Pvt. Ltd., Nagarbhavi, 5 Bengaluru - 560072, Karnataka, India. 2017.

68. Mark Zuckerberg Reveals Oculus VR GLOVES That Let You Type In Thin Air. 2017.

69. FEELREAL VR Mask \& NIRVANA VR Helmet. 2015. Feelreal.com.

70. Gloveone: The haptic glove to feel \& touch real virtual reality. NeuroDigital. 2017.

71. Gu X, Zhang Y, Sun W, et al. Dexmo: An Inexpensive and Lightweight Mechanical Exoskeleton for Motion Capture and Force Feedback in VR. 2016.

72. Manus VR. The Pinnacle of Virtual Reality Controllers. Manus-vr.com. 2015.

73. The Hands Omni: Not Just Full of Air - Virtual Reality. Virtual Reality. 2017.

74. Control VR- the Future of Virtual Reality, Animation \& more. Kickstarter. 2017. 